journal club

\title{
Hirnblutungsrisiko unter SSRI-Einnahme höher als unter Trizyklika
}

Fragestellung: Erhöht die Einnahme von selektiven SerotoninWiederaufnahmehemmern (SSRI) das Risiko spontaner intrakranieller Blutungen?

Hintergrund: Antidepressiva gehören zu den am häufigsten verwendeten Medikamenten in den Industrieländern. Sie werden nicht nur zur Behandlung von Depressionen, sondern auch in der Therapie des neuropathischen Schmerzsyndroms eingesetzt. SSRI haben unter anderem eine hemmende Wirkung auf die Thrombozytenfunktion. Seit langem ist eine Assoziation zwischen der Einnahme von SSRI und einem erhöhten Risiko für das Auftreten gastrointestinaler Blutungen bekannt. In der hier vorliegenden, populationsbezogenen retrospektiven Studie sollte untersucht werden, ob es auch einen Zusammenhang zwischen einer SSRI-Einnahme und dem Auftreten von spontanen intrakraniellen Blutungen gibt.

Patienten und Methodik: Die retrospektive Studie stützt sich auf den United Kingdom's Clinical Practice Research Datalink (CPRD). Hierbei handelt es sich um eine Datenbank, in der die Daten von rund zwölf Millionen Patienten aus 650 Praxen von Hausärzten eingegeben sind. Die Datenbank erfasst demografische Parameter, Krankheiten, Diagnosen und Symptome, Laborwerte, Arzneimittelverordnungen und Überweisungen an Spezialisten sowie Krankenhausaufnahmen.

Im Rahmen dieser Studie wurden die Daten von Patienten im Alter über 18 Jahren ausgewertet, die erstmals zwischen Januar 1995 und Juni 2014 eine Verordnung für ein Antidepressivum erhalten hatten. Erfasst wur-

Renoux C, Vahey S, Dell'Aniello $S$ et al. Association of selective serotonin reuptake inhibitors with the risk for spontaneous intracranial hemorrhage. JAMA Neurol 2017; 74: 173-80 de das Auftreten von intrakraniellen Blutungen, unter anderem parenchymatösen Hirnblutungen, Subduralhämatomen und Subarachnoidalblutungen. Für jeden Patienten, der ein Antide- pressivum verschrieben bekommen hatte, wurden bis $\mathrm{zu} 30$ Kontrollen, die bezüglich Geschlecht, Alter und Verlaufsbeobachtung gematcht waren, identifiziert.

Ergebnisse: Insgesamt wurden 1.363.990 Patienten, die erstmalig ein Antidepressivum verschrieben bekommen hatten, registriert. Davon hatten 773.364 (56,7\%) einen SSRI und 534.587 (39,2\%) ein Trizyklikum erhalten. Bei 56.039 Patienten (4,1\%) war ein Antidepressivum aus einer anderen Substanzklasse verordnet worden.

Als Kontrollen dienten 89.702 gematchte Patienten. Das mittlere Alter der Patienten bei der Antidepressiva-Erstverordnung betrug 47,9 Jahre. Mit $64 \%$ überwog der Frauenanteil. Im Verlauf einer mittleren Beobachtungszeit von 5,8 Jahren erlitten insgesamt 3.036 Patienten eine intrakranielle Blutung. Wurde das intrakranielle Blutungsrisiko zwischen Patienten verglichen, die einen SSRI einnahmen und denen, die mit trizyklischen Antidepressiva behandelt wurden, fand sich eine relative Risikoerhöhung von 1,17 zuungunsten der SSRI (95\%-Konfidenzintervall [KI]: 1,02-1,35).

In absoluten Zahlen war das Risiko einer intrakraniellen Blutung um 6,7 pro 100.000 Personenjahre erhöht. Das Risiko war in den ersten 30 Tagen der SSRI-Einnahme am höchsten (relatives Risiko [RR] 1,44; 95\%-KI 1,04-1,99). Darüber hinaus war das Risiko signifikant erhöht, wenn die Patienten begleitend mit Antikoagulanzien behandelt wurden (RR 1,73; $95 \%-\mathrm{KI}$ 0,89-3,39). Das Risiko einer intrakraniellen Blutung war dagegen nicht erhöht, wenn die Patienten eine Behandlung mit Thrombozytenfunktionshemmern erhielten.

Schlussfolgerungen: Die Einnahme von SSRI erhöhte das Risiko von intrakraniellen Blutungen im Vergleich zu Trizyklika. Im Vergleich zu Kontrollen war das Risiko vor allem in den ersten 30 Tagen der SSRI-Einnahme erhöht sowie bei den Patienten, die gleichzeitig mit oralen Antikoagulanzien behandelt wurden.

\section{- Kommentar von Hans Christoph Diener, Essen}

\section{Konsequenzen für das Verordnungsverhalten}

Diese große populationsbezogene Studie aus England hat Konsequenzen für das Verschreibungsverhalten. Patienten mit einem erhöhten Risiko für eine intrakranielle Blutung, beispielsweise mit einer Vorgeschichte einer intrakraniellen Blutung, sollten nach Möglichkeit mit Trizyklika und nicht mit SSRI behandelt werden. Die am stärksten gefährdetste Population sind Patienten, die aufgrund anderer Erkrankungen mit oralen Antikoagulanzien behandelt werden müssen. Angesichts eines um $73 \%$ erhöhten Risikos für intrakranielle Blutungen soll- ten oral antikoagulierte Patienten, wenn möglich, ebenfalls mit Trizyklika und nicht mit SSRI behandelt werden. Bei der Entscheidung für eine Behandlung mit Antidepressiva sollte allerdings immer berücksichtigt werden, dass eine effektive antidepressive Therapie einen höheren Stellenwert hat, als das relativ geringe Risiko intrakranieller Blutungen unter Antikoagulanzien. Bleiben Patienten mit schweren Depressionen unbehandelt, ist das Suizidrisiko sicher deutlich höher, als das Risiko einer intrakraniellen Blutung. 